\title{
WHEN IS THE MAXIMAL RING OF QUOTIENTS PROJECTIVE?
}

\author{
DAVID HANDELMAN
}

ABSTRACT. Let $R$ be an associative ring with 1 , and $Q$ its maximal ring of right quotients. If $r$ belongs to $R$, a right insulator for $r$ in $R$ is a finite subset of $R,\left\{r_{i}\right\}_{i=1}^{m}$, such that the right annihilator of $\left\{r r_{i} ; i=\right.$ $1, \ldots, m\}$ is zero. Then we have: If $Q$ is a projective right $R$-module, $Q$ is finitely generated; if $R$ is nonsingular, then $Q$ is projective as a right $R$-module if and only if there exists $e=e^{2}$ in $R$ such that $e R$ is injective and $e$ has a right insulator in $R$; under these circumstances, $R=Q$ if and only if $e$ has a left insulator in $R$. We prove some related results for torsionless $Q$, and give an example of a prime ring $R$ such that $Q$ is a cyclic projective right $R$-module, but $R \neq Q$.

Let $R$ be an associative ring with 1 ; $Q$ (or $Q(R)$ ) will denote the complete (or maximal) ring of right quotients of $R$ (e.g. [6, p. 94 on]). For a subset $A$ of $R$, we denote its right (left) annihilator by $A^{r}\left(A^{l}\right)$. Definitions of the terms essential, dense, singular submodule, etc. can be found in [6].

The term (right) insulator is defined in the abstract; left insulator is similarly defined. If every nonzero element of $R$ has a (right) insulator, then $R$ is (right) strongly prime [4], and is obviously prime. Strongly prime rings are studied in [8], [9] under the name ATF, but detailed descriptions are given in [4], [5].

We note that if $R$ is right strongly prime, then the right singular ideal $(Z(R))$ is zero, and if $R \subset S \subset Q(R)$, then $S$ is right strongly prime and $Q(S)=$ $Q(R)$ is simple [4, Proposition IV.1] and [9, Proposition 1.8].

$Q_{R}$ will indicate $Q$ considered as a right $R$-module. We wish to determine when $Q_{R}$ is projective, to answer a question of Viola-Prioli [9, Question 5].

Lemma 1 [6, Exercises 1, 2, p. 86]. A right $R$-module $M$ is projective if and only if there exist $\left\{f_{i}\right\}_{i \in I} \subset \operatorname{Hom}(M, R)$ such that $f_{i}(m)=0$ for almost all $i$, and there exists $\left\{m_{i}\right\}_{i \in I} \subset M$ such that $m=\Sigma_{i \in I} m_{i} f_{i}(m)$ for all $m$ in M. Further, $M$ is finitely generated projective if the $I$ can be chosen finite.

Lemma 2. If $S$ is a ring such that $R \subset S \subset Q(R)$, and $S_{R}$ is projective

Presented to the Society, April 23, 1974; received by the editors March 22, 1974 and, in revised form, June 24, 1974.

AMS (MOS) subject classifications (1970). Primary 16A08, 16A50; Secondary 16A12, 16A42, 16A52.

Key words and phrases. Ring of quotients, projective, injective, torsionless, nonsingular, prime ring, regular ring, simple ring. 
(as a right $R$-module), then $S_{R}$ is finitely generated.

Proof. Let $f \in \operatorname{Hom}\left(S_{R}, R\right)$. If $f(1)=0$, then for all $s$ in $S$,

$$
\left.f(s) \cdot s^{-1} R=f\left(s \cdot\left(s^{-1} R\right)\right)=f(1) \cdot\left(s^{-1} R\right)\right)=0 .
$$

Since $s^{-1} R$ is a dense right ideal of $R$ and $f(s) \in R$, we have $f(1)=0 \mathrm{im}$ plies $f=0$. If $I$ is the index set of the preceding lemma, set $J=\{i \in I \mid$ $\left.f_{i}(1) \neq 0\right\}$. $J$ is finite, and if $i \notin J, f_{i}=0$; so $J$ may take the place of $I$, and thus $S_{R}$ is finitely generated.

Lemma 3 (e.g. [9, Lemma 2.1]). If $E$ is an injective module and $M$ is nonsingular, then any homomorphism $f: E \rightarrow M$ splits (that is, $E \simeq \operatorname{ker} f \oplus$ $\operatorname{im} f$ via $f$, so ker $f$ and $\operatorname{im} f$ are injective).

Theorem 4. If $R$ is nonsingular, then $Q_{R}$ is projective if and only if there exists $e=e^{2} \in R$ such that $Q e Q=Q$ and $e Q \subset R$. Further, for this $e$, $R \neq Q$ if and only if $R e R \neq R$.

Proof. Suppose $Q_{R}$ is projective; by Lemma $2, Q_{R}$ is finitely generated, hence there exist $\left\{f_{i}\right\}_{i=1}^{n} \subset \operatorname{Hom}\left(Q_{R}, R\right)$ and $\left\{q_{i}\right\}_{i=1}^{n} \subset Q$, such that $q=$ $\sum_{i=1}^{n} q_{i} f_{i}(q)$ for all $q$ in $Q$. Since $Q_{R}$ is injective and $R$ is nonsingular, we may apply Lemma 3 ; thus $\operatorname{im} f_{i}$ is injective. Therefore $\operatorname{im} f_{i}=e_{i} R$ for some idempotent $e_{i}$ in $R$. Now $e_{i} Q$ is always an essential extension of $e_{i} R$; hence $e_{i} R=e_{i} Q . \Sigma e_{i} R=\Sigma e_{i} Q$ is a finitely generated right $Q$-ideal, so there exists $e^{2}=e \in Q$ such that $\Sigma e_{i} Q=e Q$, and obviously $e Q \subset R$. Finally, $1 \doteq \Sigma q_{i} f_{i}(1) \in Q e Q$, so $Q e Q=Q$.

If $e Q \subset R$ and $Q e Q=Q$, there exist $q_{i}, p_{i} \in Q$ such that $1=\Sigma q_{i} e p_{i}$. Define $f_{i}: Q_{R} \rightarrow R$ by $f_{i}(q)=e p_{i} q \in e Q \subset R$. Then, $q=\Sigma q_{i} f_{i}(q)$, so $Q$ is a finitely generated projective right $R$-module.

If $R e R \neq R$, then as $Q e Q=Q$, clearly $R \neq Q$.

If $R e R=R, R$ is Morita equivalent to a self-injective ring $e R e=e Q e$, so $R$ is self-injective, and thus $R=Q$.

Theorem 4 can be restated as: $R$ contains an injective right ideal $J$ such that End $\left(J_{R}\right)$ is Morita equivalent to $Q$, or that $R$ contains a nonzero right ideal of $Q, J$, such that $Q J=Q$.

Observe that in the proof we showed that the number of generators of $Q_{R}$ is the size of the left insulator in $Q$ chosen for $e$. We can even have the insulator of size 1 (so $Q_{R}$ is cyclic) and $R \neq Q$, as will be shown.

Lemma 5. If $A$ is a subset of $R$, then $A^{r}$ is an essential $R$-submodule of the right annihilator in $Q$ of $A$.

Proof. This may be deduced from [6, Exercise 6, p. 100].

Thus if $a$ belongs to $R$, a right insulator for $a$ in $R$ is a right insulator for $a$ in $Q$. 
A ring is a (right) F-ring if every proper finitely generated left ideal has nonzero right annihilator. Obviously, all regular rings are two-sided $F$-rings.

Lemma 6. If $R$ is a right F-ring, then $r \in R$ has a right insulator in $R$ if and only if $R r R=R$.

Proof. If $\left\{r_{i}\right\}_{i=1}^{n}$ is a right insulator for $r$, then $\sum_{i=1}^{n} R r r_{i}$ is a finitely generated left ideal with zero right annihilator. So $\Sigma R r r_{i}=R$, thus $R r R=R$. Conversely, if $1=\Sigma t_{i} r r_{i}$, then $\left\{r_{i}\right\}$ is a right insulator for $r$.

Theorem $4^{\prime}$. If $R$ is nonsingular, then $Q_{R}$ is projective if and only if $R$ contains an injective right ideal, $e R$ for some $e=e^{2} \in R$, such that $e$ has a right insulator in $R$. For this $e, R=Q$ if and only if $e$ has a left insulator in $R$.

Proof. Suppose $Q_{R}$ is projective. Then by Theorem 4, there exists $e Q=$ $e R \subset R$ such that (i) $e R$ is injective, and (ii) $Q e Q=Q$. From (ii), $e$ has a right insulator, $\left\{q_{i}\right\}$, in $Q$; clearly $\left\{e q_{i}\right\}$ is also a right insulator for $e$, and as $e Q \subset R_{3}$. it is an insulator for $e$ in $R$.

Assume the converse condition. Since $e R$ is injective, $e R=e Q$ (as in the proof of Theorem 4); since $e$ has a right insulator in $R$, by Lemma 5 , it has a right insulator in $Q$. Now $Q$ is regular, so by Lemma $6, Q e Q=Q$; thus $e$ satisfies the conditions of Theorem 4 and $Q_{R}$ is projective.

If this $e$ has a left insulator in $r,\left\{r_{i}\right\}$, then $\Sigma r_{i} e R=\Sigma r_{i} e Q$ is a finitely generated ideal of the regular ring $Q$, so there exists $f^{2}=f \in Q$ such that $f Q=\Sigma r_{i} e Q$. Clearly, $f Q \subset R$, and as $\left\{r_{i} e\right\}^{1}=(0)$, we must have $f^{1}=(0)$; thus $f=1$, and $Q(=f Q)=R$.

If $R=Q, R$ is regular, so if $e$ has a right insulator, by Lemma 6 , it has a left insulator.

Corollary 7. If $Q$ is simple, then $Q_{R}$ is projective and $R \neq Q$ if and only if there exists an injective right ideal of $R, J$, such that $(0) \neq R J \neq R$.

For instance if $R$ is right strongly prime, $Q$ is simple. Necessary and sufficient conditions for $Q$ to be simple will be given (with examples) in [3].

Corollary 8. If $Q_{R}$ is projective and $R$ satisfies any one of the follow. ing conditions:

(i) $R$ is left strongly prime and right nonsingular,

(ii) $R$ is a right F-ring and right nonsingular,

(iii) $R$ is commutative semiprime, then $R=Q$.

Proof. (i) is immediate from Theorem $4^{\prime}$.

(ii) By Lemma 6, if $e \in R$ has a right insulator, it also has a left insulator. 
(iii) For a commutative ring, semiprime is equivalent to nonsingular.

Condition (ii) above generalizes Theorem 2.1 of [10].

Corollary 9. If $Z(R)=(0)$ and $Q_{R}$ is a projective module, and $R \subset S \subset$ $Q$, then $Q_{S}$ is projective.

Proof. Clearly $Q(R)=Q(S)$; as $e R=e Q$, we have $e S=e Q$.

To obtain interesting examples of $Q_{R}$ projective, we consider the following

Lemma 10. If $T$ is an overring of $R$, and $a \in R$ has no left insulator in $R$, then $a$ has no left insulator in $S=\langle a T, R\rangle$, the subring of $T$ generated by $a T$ and $R$.

Proof. Since $a T$ is a right $R$-module, every element of $S$ can be written in the form: $\Sigma r_{i} a t_{i}+r ; t_{i} \in T ; r, r_{i} \in R$. Suppose that $\left\{f_{k}=\Sigma{ }_{j k} r_{j k} a t_{j k}+r_{k}\right\}$ is a finite subset of $S$. Since $a$ has no left insulator in $R$, the finite set $\left\{r_{k}, r_{j k}\right\}_{j k, k}$ is not a left insulator for $a$; hence there exists nonzero $u$ in $R$ such that $u r_{k} a=u r_{j k} a=0$ for all $k, j k$. It is clear that $u f_{k} a=0$ for all $k$, so $\left\{f_{k}\right\}$ is not a left insulator for $a$ in $S$.

Corollary 11. If $a \in R$ has no left insulator in $R$, and $Q$ is simple, then the ring $S=\langle a Q, R\rangle$ has $Q(S)=Q, S \neq Q$, and if a is nonzero, $Q_{S}$ is a finite. ly generated projective right $S$-module. Further if $R$ is prime (right strongly prime) then $S$ is prime (right strongly prime).

Proof. Follows immediately from Corollary 7 and the preceding lemma.

If $Q$ is simple self-injective and $e=e^{2} \in Q$, then $R=e Q+Q(1-e)$ has $Q=Q(R)$ (both on the right and the left) and $Q$ is projective. $e Q(1-e$ ) is a nilpotent ideal of $R$. If, in particular, $Q=M_{2} D$ where $D$ is a division ring, then we obtain the overworked example:

$$
R=\left\{\left[\begin{array}{ll}
a & 0 \\
b & c
\end{array}\right] \mid a, b, c, \in D\right\}
$$

We now construct a right strongly prime (and hence right nonsingular prime) ring, $S$, such that $Q(S)$ is a cyclic projective right $S$-module. This answers in the negative two questions of Viola-Prioli [9, Questions 5 and 61 .

Let $F$ be a field, and $D=F[x, y]$, the free noncommuting algebra on two variables. $D$ is a domain, so is right strongly prime. Further $D \times D=$ $\bigoplus_{i=0}^{\infty} y^{i} x D \simeq \bigoplus^{\boldsymbol{\aleph}_{0}} D_{D}$; i.e., $D x D$ is a countably generated free right $D$. module. Now, $D x D^{l}=(0)$, so the map (of rings)

$$
D \rightarrow \operatorname{End}\left(D x D_{D}\right), \quad r \mapsto \hat{r} ; \quad \hat{r}(d)=r d
$$

is a monomorphism, and $R=\operatorname{End}(D x D)$ is an essential extension of $D$ as a 
right $D$-module, so $D \subset R \subset Q(D)=Q(R)$. Hence $R$ is right strongly prime. But $R \simeq \operatorname{End}\left(\bigoplus D_{D}\right)$, the ring of $\boldsymbol{\kappa}_{0} \times \boldsymbol{\kappa}_{0}$ matrices over $D$, with finitely many nonzero entries in each column. We show $R$ has a proper torsion ideal [7, Chapter 2] on the left.

Let $r \in R$, write $r=\left(r_{i j}\right)$, where $r_{i j}$ is the $i, j$ entry on the $\boldsymbol{\kappa}_{0} \times \boldsymbol{\kappa}_{0}$ matrix that $r$ represents, and let $e_{i}$ be the matrix with a 1 in the $i, i$ position and 0 elsewhere. Then $K=\bigoplus_{i=1}^{\infty} e_{i} R$ is a two-sided ideal and $K=$ $\left\{r \in R \mid\right.$ there exists $N_{r}$ such that $i \geq N_{r}$ implies $\left.r_{i j}=0\right\}$ (each element of $K$ contains only finitely many rows with nonzero entries).

Claim. $R / K$ is flat as a right $R \cdot$ module.

In view of the proof of [7, Proposition 2.3], it suffices to show, given $k \in K$, there exists $k^{\prime}$ e $K$ such that $k^{\prime} k=k$. If $k=\left(k_{i j}\right) \in K$, there exists $N_{k}$ such that $i \geq N_{k}$ implies $k_{i j}=0$. Set $k^{\prime}=\sum_{i=1}^{N_{k}} e_{i} \in K$; then $k^{\prime} k=k$.

Since $R / K$ is right flat, it follows from [7, Proposition 2.3], that $K$ is a left torsion ideal. Since the corresponding torsion theory is closed under (in particular) submodules, direct sums and factors, it follows that no element of a proper torsion ideal may have a left insulator; in particular, $e_{1}$ has no left insulator in $R$. Since $D$ is a domain and thus strongly prime, $Q(D)=$ $Q(R)$ is simple; set $S=\left\langle e_{1} Q, R\right\rangle$. By Corollary $11, Q(S)=Q(D)$ is a finitely generated projective right $S$-module, $S$ is right strongly prime (hence prime nonsingular) and $S \neq Q(S)$.

We note that since $D$ is a domain, every element in $Q(D)=Q(S)$ has a one-element insulator: choose nonzero $q$ in $Q$; there exists $d \in D$ such that $q d$ is nonzero and belongs to $D$. Therefore $(q d)^{r}$ is zero, so the right annihilator in $Q$ of $q d$ is zero by Lemma 5. So $\{d\}$ is the desired insulator. Since $Q$ is regular, there exists $u, v \in Q$ such that $u e{ }_{1} v=1$; hence $Q=$ $u Q=u e_{1} Q=u e_{1} S$; thus $Q$ is a cyclic right $S$-module.

A module $M$ is torsionless if there exists a monomorphism $M \rightarrow \pi R$. Obviously a projective module is torsionless.

In [1], conditions for a nonsingular semiprime ring to have $Q$ torsionless are given. As noted in [1], for any ring, the sum of right ideals which are injective as right modules is a two-sided ideal. We say a two-sided ideal is right insulated if it contains a finite set whose right annihilator is zero.

Theorem 12. If $Z(R)=(0)$, then $Q_{R}$ is torsionless if and only if the sum of the injective right ideals is a faithful right $R$-module. Further $R=Q$ if and only if the sum of the injective right ideals is left insulated.

Proof. As $Q_{R}$ is torsionless, for all $q$ in $Q$, there exists $f_{q} \in \operatorname{Hom}\left(Q_{R}, R\right)$ such that $f_{q}(q) \neq 0$. By Lemma $3, f_{q}(Q)$ is an injective right ideal, and $\Sigma_{q \in Q} f_{q}(Q)$ is obviously faithful.

If $\left\{e_{j} R\right\}$ are injective and their sum is faithful, then $\pi e_{j} R$ is a faithful module. Hence there exists a monomorphism $R \rightarrow \pi e_{j} R$; as $\pi e_{j} R$ is injective 
and $Q$ is the injective hull of $R$, there exists a monomorphism $Q \rightarrow \pi e{ }_{j} R \subset \pi R$.

If $R=Q, 1$ belongs to the sum of the injective right ideals.

If $R \neq Q$, proceed as in Theorem $4^{\prime}$.

Corollary 13. For $Z(R)=(0), Q_{R}$ is torsionless if $R$ contains a faithful injective right ideal.

The condition of Corollary 13 is sufficient but not necessary: Put $Q=$ $\pi F_{j}$ (an infinite product of fields), and $R=\left\langle\bigoplus F_{j}, 1\right\rangle$. Then $Q_{R}=Q(R)_{R}$ is torsionless, but $R$ contains no faithful injective right ideals.

Corollary 14. If $Q$ is prime regular, then $Q_{R}$ is torsionless if and only if $R$ contains a nonzero injective right ideal.

Corollary 15. If $Z(R)=(0)$, and $Q_{R}$ is torsionless, then $Q$ is an essential extension of $R$ as a left $R$-module.

Proof. Let $K$ be the sum of the injective right ideals of $R$, and $q$ a nonzero element of $Q$. Then $K q \neq(0)$ as $K$ is faithful, and $K q \subset K$ since any injective right ideal of $R$ is of the form $e R=e Q$. Hence $(0) \neq K q \subset K \subset R$.

Corollary 16 [1, Theorem 4]. If $R$ is semiprime, right nonsingular, then $Q_{R}$ is torsionless if and only if the sum of the injective right ideals is essential as a right ideal.

Proof. In a semiprime ring, a two-sided ideal is (right) faithful if and only if it is essential as a right ideal.

\section{REFERENCES}

1. E. P. Armendariz, A note on semiprime rings with torsionless injective envelopes, Canad. Math. Bull. 16 (1973), 429-431.

2. C. C. Faith, Lectures on injective modules and quotient rings, Lecture Notes in Math., no. 49, Springer-Verlag, Berlin and New York, 1967. MR 37 \#2791.

3. K. Goodearl and D. Handelman, Simple self-injective rings (to appear).

4. D. Handelman and J. Lawrence, Strongly prime rings, Trans. Amer. Math. Soc. 211 (1975), 209-223.

5. K. Goodearl, D. Handelman and J. Lawrence, Strongly prime, completely torsion-free and simple self-injective rings, Carleton Mathematical Series, no. 109, 1974.

6. J. Lambek, Lectures on rings and modules, Blaisdell, Waltham, Mass., 1966. MR $34 \# 5857$.

7. - Torsion theories, additive semantics, and rings of quotients, Lecture Notes in Math., vol. 177, Springer-Verlag, Berlin and New York, 1971. MR 44\#1685.

8. R. A. Rubin, A bsolutely torsion-free rings, Pacific J. Math. 46 (1973), 503514.

9. J. Viola-Prioli, On absolutely torsion-free rings and kernel functors, Ph.D. Dissertation, Rutgers University, 1973.

10. V. C. Cateforis, On regular self-injective rings, Pacific J. Math. 30 (1969), 39-45. MR 40 \#1432.

DEPARTMENT OF MATHEMATICS, MCGILL UNIVERSITY, P.O. B OX 6070, STATION A, MONTREAL, QUEBEC, CANADA H3C 3G1

Current address: Mathematisches Institut, Universität Giessen, 6300 Giessen, West Germany 